\title{
ARTICLE OPEN \\ Circadian misalignment affects sleep and medication use before and during spaceflight
}

\author{
Erin E Flynn-Evans ${ }^{1,2,3}$, Laura K Barger ${ }^{1,2}$, Alan A Kubey ${ }^{1}$, Jason P Sullivan ${ }^{1}$ and Charles A Czeisler ${ }^{1,2}$
}

Sleep deficiency and the use of sleep-promoting medication are prevalent during spaceflight. Operations frequently dictate work during the biological night and sleep during the biological day, which contribute to circadian misalignment. We investigated whether circadian misalignment was associated with adverse sleep outcomes before (preflight) and during spaceflight missions aboard the International Space Station (ISS). Actigraphy and photometry data for 21 astronauts were collected over 3,248 days of long-duration spaceflight on the ISS and 11 days prior to launch ( $n=231$ days). Sleep logs, collected one out of every 3 weeks in flight and daily on Earth, were used to determine medication use and subjective ratings of sleep quality. Actigraphy and photometry data were processed using Circadian Performance Simulation Software to calculate the estimated endogenous circadian temperature minimum. Sleep episodes were classified as aligned or misaligned relative to the estimated endogenous circadian temperature minimum. Mixed-effects regression models accounting for repeated measures were computed by data collection interval (preflight, flight) and circadian alignment status. The estimated endogenous circadian temperature minimum occurred outside sleep episodes on $13 \%$ of sleep episodes during preflight and on $19 \%$ of sleep episodes during spaceflight. The mean sleep duration in low-Earth orbit on the ISS was $6.4 \pm 1.2 \mathrm{~h}$ during aligned and $5.4 \pm 1.4 \mathrm{~h}(P<0.01)$ during misaligned sleep episodes. During aligned sleep episodes, astronauts rated their sleep quality as significantly better than during misaligned sleep episodes $(66.8 \pm 17.7$ vs. $60.2 \pm 21.0, P<0.01)$. Sleep-promoting medication use was significantly higher during misaligned $(24 \%)$ compared with aligned $(11 \%)$ sleep episodes $(P<0.01)$. Use of any medication was significantly higher on days when sleep episodes were misaligned (63\%) compared with when sleep episodes were aligned $(49 \% ; P<0.01)$. Circadian misalignment is associated with sleep deficiency and increased medication use during spaceflight. These findings suggest that there is an immediate need to deploy and assess effective countermeasures to minimize circadian misalignment and consequent adverse sleep outcomes both before and during spaceflight.

npj Microgravity (2016) 2, 15019; doi:10.1038/npjmgrav.2015.19; published online 7 January 2016

\section{INTRODUCTION}

It has been demonstrated that astronauts sleep less during spaceflight than they do on Earth; ${ }^{1-5}$ however, the causes of shortened sleep during spaceflight are not well understood. Sleep disturbances have, in part, been attributed to environmental factors, such as noise and uncomfortable temperatures. ${ }^{1}$ Microgravity and hypercapnia have also been postulated to cause sleep deficiency. ${ }^{4}$ We hypothesize that circadian misalignment may be a significant contributor to the sleep deficiency observed in space.

The primary synchronizer of human circadian rhythms to the 24-h day is light exposure. In the absence of exposure to light of sufficient intensity, timing, duration, and spectral composition, human circadian pacemakers will revert to oscillating at their intrinsic period, which is slightly longer than $24 \mathrm{~h}$ for most individuals. $^{6}$ This can cause circadian misalignment between the biological day and 24-h social day. Circadian misalignment leads to shortened sleep duration when sleep is attempted during the biological day as well as cognitive impairment associated with being awake during the biological night. ${ }^{7}$

In low-Earth orbit, the period of the solar light-dark cycle is 90 min, which is far too short for circadian entrainment in humans. Adherence to a rigorously imposed artificial light-dark cycle with a 24-h period could facilitate circadian entrainment during low-Earth orbit; however, the imposition of 6- to 12-h phase shifts in the timing of the crew members' sleep-wake cycles for routine docking and other maneuvers; ${ }^{8}$ exposure to the 90 -min light-dark cycle from the skylight "cupola" and other windows on the International Space Station (ISS), which has historically occurred even during scheduled sleep episodes; ${ }^{9}$ and exposure to light from personal light-emitting eReaders ${ }^{10}$ can each induce circadian misalignment. During the spaceflight missions aboard the Russian Mir, where operations followed a 24-h day, a drift in circadian phase was observed during 100 days of spaceflight in a single astronaut ${ }^{11}$ and in three out of four astronauts studied during short-duration missions on Mir and Soyuz. ${ }^{3}$ Similarly, studies on the Space Shuttle missions STS-90 and STS-95 showed that astronauts failed to entrain to a phase advance schedule and that ambient light intensity was very low, with brief, significant intrusions of the 90 -min orbital solar light-dark cycle. ${ }^{2}$

Although spaceflight operations have changed since the Mir missions, astronauts on the ISS are often required to maintain wakefulness at adverse circadian phases due to mission demands. Space Shuttle and Soyuz launch windows are limited to narrow bands of time each day to ensure that the vehicles achieve a

\footnotetext{
${ }^{1}$ Department of Medicine, Division of Sleep and Circadian Disorders, Brigham and Women's Hospital, Boston, MA, USA; ${ }^{2}$ Department of Medicine, Division of Sleep Medicine, Harvard Medical School, Boston, MA, USA and ${ }^{3}$ Exploration Technology Directorate, Fatigue Countermeasures Laboratory, Human Systems Integration Division, NASA Ames Research Center, Moffett Field, CA, USA.

Correspondence: EE Flynn-Evans (erin.e.flynn-evans@nasa.gov)

Received 8 May 2015; revised 28 August 2015; accepted 19 October 2015
} 
Table 1. Baseline characteristics among study participants

\begin{tabular}{lc}
\hline & Mean (s.d.) \\
\hline$n$ & 21 \\
Age (years) & $46.7(3.9)$ \\
Days in flight & $155(39)$ \\
& $n(\%)$ \\
Male & $15(71)$ \\
Launch on Soyuz & $14(67)$ \\
Launch on Shuttle & $7(33)$ \\
\hline
\end{tabular}

suitable orbit for a given mission. Astronauts need to awaken at least $5 \mathrm{~h}$ prior to the appointed launch time, which determines scheduled sleep times throughout the flight; therefore, an 0800 hours launch requires a middle-of-the night awakening and considerable circadian adjustment. In the pre-launch quarantine week prior to flight at Kennedy Space Center, astronauts are typically exposed to bright light in the astronaut crew quarters to facilitate circadian adaptation to the launch schedule. ${ }^{12}$ During spaceflight, vehicle docking and undocking with ISS can occur at any time during the day or night. In order to prepare for these events, ISS astronauts are often required to "slam-shift," whereby they suddenly are required to sleep at a time many hours before or after their nominal bedtime in order to have scheduled wakefulness coincide with mission events. These abrupt shifts in the imposed sleep-wake schedule can induce circadian misalignment.

Circadian phase has been previously studied in only a small number of astronauts. Using actigraphy and photometry data from the largest study of sleep ever conducted during spaceflight and a model to estimate circadian phase, we aimed to quantify the frequency and timing of circadian misalignment during the preflight interval and relative to mission critical events during long-duration spaceflight on the ISS. We further aimed to assess the impact of circadian misalignment on sleep duration, quality, and medication use.

\section{RESULTS}

The present analysis includes data for 21 missions aboard the ISS encompassing 3,248 days of spaceflight, of which 2,864 24-h intervals of actigraphy and photometry met the inclusion criteria for analysis using CPSS. Each participant ( $n=21,15$ males) contributed an average $155( \pm 39)$ days of data in flight (Table 1$)$, with an average of 137 24-h intervals of actigraphy and photometry that met inclusion criteria for analysis in CPSS (range 59-214 days). Of these, the sleep diary-including subjective sleep reports, sleep quality ratings and medication use-was collected for 863 24-h intervals. We collected data on 231 days during the preflight interval, of which 206 24-h intervals of actigraphy and photometry met the inclusion criteria for analysis using CPSS. Table 2 shows the sleep characteristics for the preflight and spaceflight data collection.

During the preflight interval, we found that the estimated endogenous circadian temperature minimum was aligned during 180 sleep episodes ( $87 \%$ ) and there were 26 sleep episodes when the estimated endogenous circadian temperature minimum was misaligned (13\%). During the preflight interval, astronauts slept, on average, significantly longer when the estimated endogenous circadian temperature minimum was aligned $(6.0 \pm 1.2 \mathrm{~h})$, compared with when the estimated endogenous circadian temperature minimum was misaligned $(5.5 \pm 1.8 \mathrm{~h} ; P=0.0016)$. We found a statistically significant difference in the frequency of circadian misalignment during the preflight interval when astronauts launched from Baikonur, Kazakhstan, when the estimated endogenous circadian temperature minimum was circadian
Table 2. Sleep characteristics among study participants by data collection period

\begin{tabular}{lcc}
\hline & L-11 mean (s.d.) & Flight mean (s.d.) \\
\hline Actigraphy sleep duration $(\mathrm{h})$ & $5.9(0.9)$ & $6.1(0.7)$ \\
Subjective sleep duration $(\mathrm{h})$ & $6.3(0.8)$ & $6.5(0.7)$ \\
Latency $(\mathrm{m})$ & $14.4(9.5)$ & $13.7(10.1)$ \\
Number of awakenings & $1.3(0.8)$ & $1.5(1.0)$ \\
Sleep efficiency* & $87 \%(5 \%)$ & $89 \%(5 \%)$ \\
Sleep quality & $62.3(15.6)$ & $66.5(13.4)$ \\
Alertness & $56.0(19.5)$ & $57.7(18.7)$ \\
\hline
\end{tabular}

*The sleep efficiency was calculated from actigraphy as the time scored as sleep/the sleep period time.

misaligned during $8 \%$ of sleep episodes, compared with $21 \%$ of sleep episodes when astronauts launched from Cape Canaveral, FL, USA $(P=0.004)$. We found no significant difference in sleep duration between astronauts who traveled to ISS on Soyuz during the preflight interval compared to those who traveled on the Space Shuttle ( $5.87 \mathrm{~h} \pm 1.25$ vs. $5.97 \mathrm{~h} \pm 1.43, P=0.67$; respectively). During preflight, participants reported using sleep medication on $15 \%$ of both circadian aligned and misaligned sleep episodes $(P=0.94)$. No astronauts reported using prescribed alertness medications during the preflight interval. Astronauts reported preflight use of any type of medication on $77 \%$ of days when the sleep episodes were circadian misaligned compared with $53 \%$ of days when the sleep episode was circadian aligned $(P=0.02)$. Caffeine use was reported on $91 \%$ of days during the preflight interval and there was no difference in the use of caffeine on days where the estimated endogenous circadian temperature minimum was estimated to be circadian aligned versus misaligned ( $90 \%$ vs. $100 \%$, respectively; $P=0.23$ ).

During spaceflight, the endogenous circadian temperature minimum was estimated to be circadian aligned on 2,308 sleep episodes in flight (81\%) and circadian misaligned on 556 sleep episodes (19\%). Representative plots from three astronauts showing the estimated endogenous circadian temperature minimum and sleep episode timing each day are presented in Figure 1. Table 3 shows in-flight sleep outcomes compared between aligned and misaligned sleep episodes. The mean sleep duration for circadian aligned sleep episodes was significantly longer $(6.4 \pm 1.2 \mathrm{~h})$ compared with circadian misaligned sleep episodes $(5.4 \pm 1.4 \mathrm{~h} ; P<0.0001)$. Participants' sleep episodes were more likely to be misaligned when a vehicle was docked with ISS (23\%) compared with when no vehicle was docked (18\%; $P=0.05$ ). Similarly, sleep duration was shorter when a vehicle was docked with the ISS $(6.0 \pm 1.1 \mathrm{~h}$ docked vs. $6.2 \pm 1.3 \mathrm{~h}$ undocked, $P<0.0001)$. In addition, $29 \%$ of sleep episodes before an EVA were misaligned, compared with $19 \%$ of sleep episodes that did not precede this critical mission task $(P=0.19)$.

During spaceflight, astronauts reported using sleep medication on $11 \%$ of circadian aligned sleep episodes compared with $24 \%$ of circadian misaligned sleep episodes $(P=0.0002)$. Prescribed alertness medications were reported $<1 \%$ of the time for both aligned and misaligned 24-h intervals. They also reported using any medication on $49 \%$ of circadian aligned sleep episodes compared with $63 \%$ of circadian misaligned sleep episodes $(P=0.008)$. Astronauts reported using caffeine $91 \%$ of days when sleep episodes were misaligned and $92 \%$ of days when sleep episodes were aligned $(P=0.68)$.

\section{DISCUSSION}

Circadian misalignment of scheduled sleep episodes was common both before and during spaceflight, occurring approximately once out of every five sleep episodes on the ISS. When crewmembers' 

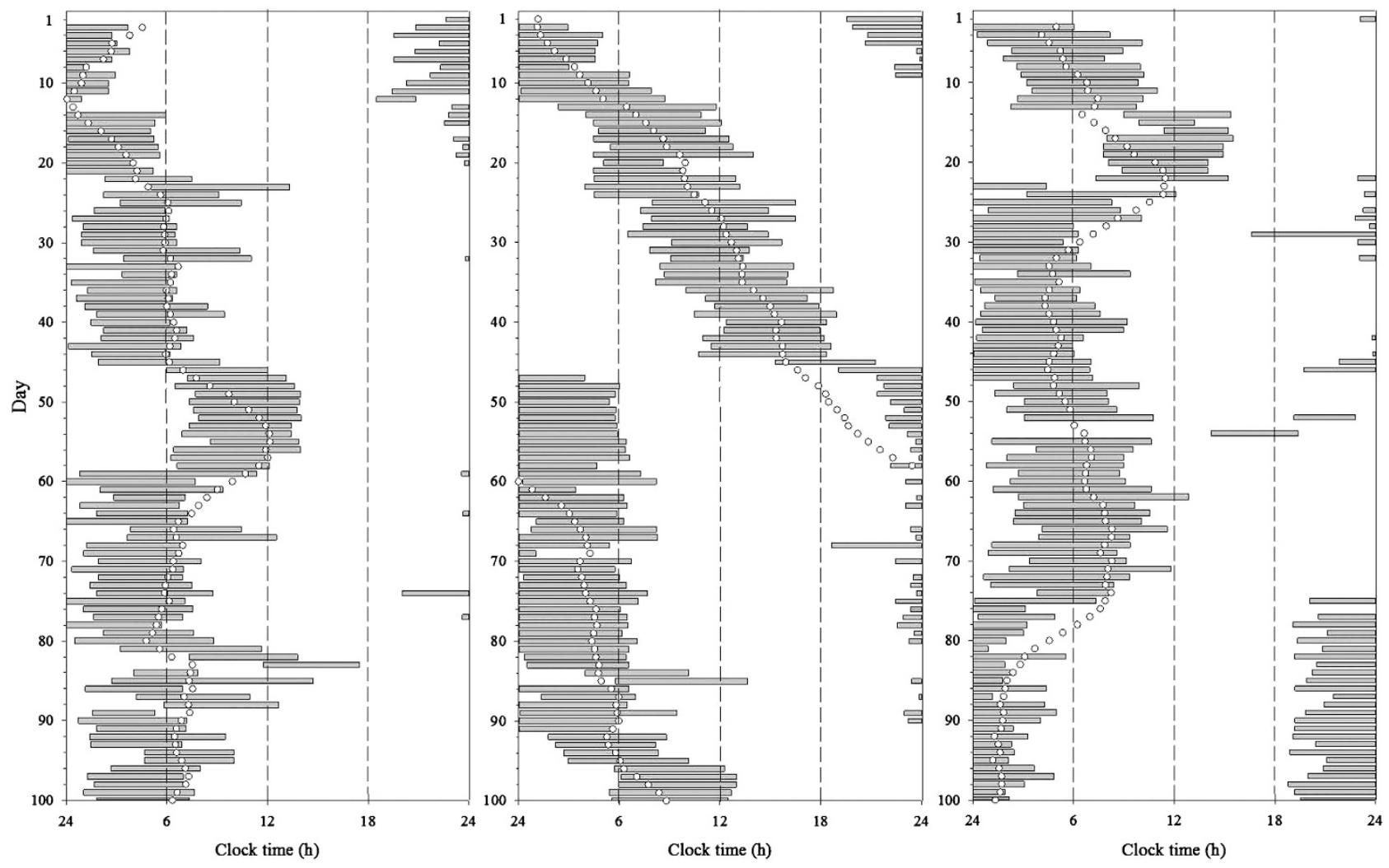

Figure 1. Representative raster plots for three astronauts during spaceflight. Plots show sleep (black boxes) and circadian temperature minimum estimates (circles) derived from actigraphy over 100 days of data collection during spaceflight. Day 1 begins in the upper left corner. Data are plotted by clock time as indicated on the lower axis.

Table 3. Sleep characteristics during nights in flight where the temperature minimum was predicted to occur during the sleep episode compared with nights predicted to occur outside the sleep episode

\begin{tabular}{|c|c|c|c|}
\hline & Aligned & Misaligned & \\
\hline & Mean (s.d.) & Mean (s.d.) & P-value \\
\hline Actigraphy sleep duration (h) & $6.4(1.2)$ & $5.4(1.4)$ & $<0.0001$ \\
\hline Latency $(\mathrm{m})^{\mathrm{a}}$ & $10.3(15.0)$ & $13.2(25.2)$ & 0.26 \\
\hline Number of awakenings ${ }^{a}$ & $1.7(1.9)$ & $1.7(1.7)$ & 0.38 \\
\hline Actigraphy sleep efficiency & $89 \%(7 \%)$ & $90 \%(7 \%)$ & 0.26 \\
\hline Sleep quality ${ }^{\mathrm{a}}$ & $66.8(17.7)$ & $60.2(21.1)$ & 0.01 \\
\hline Alertness $^{\mathrm{a}}$ & $57.9(21.7)$ & $53.6(21.5)$ & 0.13 \\
\hline
\end{tabular}

sleep episodes were misaligned, they slept nearly $1 \mathrm{~h}$ less per night than when their sleep episodes were aligned. They also reported experiencing poorer sleep quality during sleep episodes that were misaligned. Of importance, astronauts were more likely to be circadian misaligned during critical operations, such as when a vehicle was docked with the ISS. When their sleep episodes were circadian misaligned, astronauts were more likely to use both sleep medications at night and medication of any kind during the day, suggesting that the impact of circadian misalignment extends beyond sleep outcomes.

In this report, we demonstrate a novel approach for longitudinal estimation of circadian phase that enables evaluation throughout a spaceflight mission, without the need for obtrusive specimen collection. Circadian misalignment has been difficult to quantify during spaceflight, because measurement of circadian phase typically requires frequent collection of biomarkers of circadian phase, such as blood, saliva or urine, or core body temperature; these recurrent measures are rarely feasible during operational spaceflight. We were able to model circadian phase using activity and light inputs from non-invasive wrist actigraphy and photometry, which are widely used by both clinicians and consumers. Our method of circadian phase estimation has many terrestrial applications whenever circadian phase information would be useful in guiding operational or treatment decisions. Given the high prevalence of shift work in the general population, ${ }^{13}$ this model could be used to assess the magnitude of circadian misalignment in other industries, such as in aviation, medicine and first responders, in which maintaining peak levels of alertness and performance is essential. Incorporating circadian modeling into schedule design in these occupations could enhance operational decisions and assist managers in making fitness-for-duty recommendations. This model could also be used to guide countermeasure use to mitigate the impact of circadian misalignment on worker productivity and safety.

Although circadian misalignment is prevalent among astronauts and the general population, the health consequences of such activities are only now beginning to be understood. Circadian disruption has been linked to both immediate and long-term adverse health consequences, ${ }^{14}$ such as impaired glucose regulation and metabolism, ${ }^{15}$ increased risk of coronary heart disease, ${ }^{16}$ and increased breast ${ }^{17}$ and prostate cancer risk. ${ }^{18}$ Short sleep duration, circadian misalignment, and long work hours have also been repeatedly linked to cognitive impairment, including attentional failures, ${ }^{19}$ actual and near-miss medical errors, ${ }^{20}$ fatigue-related motor vehicle crashes ${ }^{21}$ and rare, 
but major catastrophic events such as the Exxon Valdez oil spill, ${ }^{22}$ and the Space Shuttle Challenger Accident. ${ }^{23}$ Critical and potentially dangerous mission tasks are often scheduled during and following vehicle docking. This is when we found that circadian misalignment and its associated sleep loss and increased medication use are most likely to occur. This is of particular concern given that sleep deficiency contributed to the most serious collision that has occurred during spaceflight, during the docking of the Progress re-supply ship and the Mir Space Station in 1997. ${ }^{24}$

The typical safeguard against error related to circadian misalignment and sleep loss in the shift working industry is the use of redundant systems, such as automated prescription checks to intercept medication errors in hospitals and standardized checklists and pilot-co-pilot communication in aviation. Despite the prevalence of such coping systems, failures associated with circadian misalignment still occur, such as during the Asiana Airlines crash in 2013 during an approach to the San Francisco International Airport, which coincided approximately with the circadian nadir for the four pilots aboard the aircraft, leading to impaired decision-making and loss of situational awareness. ${ }^{25}$ Currently, National Aeronautics and Space Administration employs many back-up systems that likely prevent major failures on the ISS. For example, during docking, robotics operations, and EVAs, the primary operator of the robotic arm is backed up by a mission specialist, who may identify and prevent gross errors in action. In addition, the ground-based mission control support staff remain in near-continuous communication with orbiting astronauts and detailed checklists are in place to guide mission operations. Unfortunately, the present and prior studies indicate that those astronauts and support crew working in back-up roles are likely circadian misaligned and sleep-deprived as well, $^{26,27}$ leaving each level of back-up vulnerable to potential cognitive failure. Chronic circadian misalignment also interferes with learning during training. ${ }^{28}$

During operations extending beyond low-Earth orbit, e.g., on future manned missions to Mars and beyond, such back-up systems may not be feasible due to communication delays. Studies examining adaptation to the non-24-hour Martian day $^{29}$ and simulated missions to Mars have shown that circadian misalignment is likely to occur for some parts or all of the duration of a mission in most astronauts, ${ }^{30}$ a finding that highlights the need to develop effective countermeasures to facilitate circadian entrainment during space exploration.

In addition to sleep deficiency and circadian misalignment, cognitive impairment arising from the prevalent use of sleep medications remains a concern. The use of sleep and wakepromoting medications to improve sleep and mitigate fatigue may be a reasonable countermeasure when taken under the guidance of a flight surgeon for specific situations when sleep timing must be shifted abruptly, requiring an astronaut to remain awake for an extended duration shift or during an episode of circadian misalignment. However, at the present time, sleep medication use during spaceflight is $10-20$ times higher than that consumed by the general population. ${ }^{31}$ This suggests that either sleep medications are ineffective as countermeasures or the use of sleep medication during spaceflight may be high due to the lack of other effective countermeasures available to prevent circadian misalignment during spaceflight. We found that astronauts took sleep medication more often when they were misaligned. This suggests that astronauts recognized the importance of sleep and took sleep medications more commonly when they were unable to obtain adequate sleep. Of importance, traditional hypnotics do not shift the circadian rhythm. This means that if an astronaut were scheduled to work in a misaligned state, his/her circadian rhythm would not shift into circadian alignment with the imposed schedule in the absence of countermeasures such as light or chronobiotics such as melatonin or melatonin analogs. It may be possible to combine lighting, sleep, and/or wake- promoting countermeasures in order to accelerate circadian alignment; however, there has only been one randomized controlled trial conducted to evaluate the efficacy of melatonin use during spaceflight and that report found that $0.3 \mathrm{mg}$ of melatonin resulted in a shorter sleep latency, but no change in any other sleep parameters. ${ }^{2}$

We also found that astronauts were more likely to take other medications when they were misaligned. This suggests that circadian misalignment may enhance the symptoms of other medical conditions ${ }^{32}$ and pain, ${ }^{33}$ prompting a general increase in self-selected medication use. Our data suggest that circadian misalignment may also contribute to sleep medication use among shiftworkers and those with insomnia. Based on our findings and that of others, targeted measures to facilitate circadian alignment among these populations could increase sleep duration and decrease the need for medication use, increasing sleep duration by up to $2 \mathrm{~h}$ per day. ${ }^{34}$

The primary countermeasure to facilitate circadian alignment to altered work schedules is appropriately timed exposure to both light and darkness. ${ }^{35}$ Lighting schemes that incorporate shortwavelength-enriched light are highly effective in eliciting circadian phase shifts and in producing acute alerting effects in laboratory studies. ${ }^{36-38}$ During the preflight data collection block for astronauts flying aboard the Space Shuttle, bright-light exposure was available as a countermeasure to facilitate circadian adjustment to the preflight schedule that required a change in the sleep-wake times. Given that, we observed a higher prevalence of misalignment among astronauts launching from Kennedy Space Center compared to those launching from Baikonur. We hypothesize that preflight workload prevented astronauts from benefiting from this countermeasure, but this would require further study. Prior studies have demonstrated that such a preflight light exposure regime is sufficient to phase shift most astronauts up to $12 \mathrm{~h} .{ }^{12,39,40}$ Similar lighting schemes have been integrated into some terrestrial settings where 24-h operations are required and have led to improvements in worker alertness and performance. ${ }^{41}$ New lighting systems are being deployed to the ISS in an effort to improve circadian entrainment and alertness among astronauts during critical tasks. ${ }^{42-44}$ Scheduling countermeasures to facilitate circadian alignment prior to large, rapid shifts in the sleep/wake cycle (also known as "slam shifts"), coupled with enhanced lighting schemes on ISS, need to be evaluated to determine whether they are sufficient to reduce the number of misaligned sleep episodes in space.

Although this is the largest cohort of astronauts assessed for circadian misalignment during spaceflight, our study is not without limitation. We were only able to collect sleep, activity, and illuminance levels via actigraphy, photometry, and sleep logs. As such, we estimated circadian phase using a mathematical model developed from laboratory data. ${ }^{45}$ We asked the astronauts to keep the actiwatch uncovered and to wear the actiwatch at all possible times; however, given that this study was conducted in a spaceflight environment, we did not control astronaut behavior and we have no information regarding when the actiwatch light sensor was covered by a shirtsleeve. In cases where the light sensor was covered, the model input would be less accurate. In order to account for this possible effect, we binned light data in $1 \mathrm{~h}$ increments. We also considered circadian phase to be aligned when the circadian nadir was predicted to fall anytime within the astronaut's sleep episode; however, abnormal timing of circadian phase within a sleep episode has been shown to be associated with decrements in performance and insomnia. ${ }^{46}$ As a result, our definition of alignment likely included some individuals who experienced abnormal entrainment. We would expect that this would attenuate our findings and that more precise estimation of circadian phase would likely reveal a higher prevalence of circadian misalignment. 
In summary, we found that astronauts completing longduration missions aboard ISS experience circadian misalignment $\sim 20 \%$ of days in flight, resulting in $\sim 1 \mathrm{~h}$ loss in sleep per night. We also found that astronauts take sleep medications and medication of any kind more frequently during circadian misalignment. We further find that circadian misalignment occurs more often when a vehicle is docked with ISS. Further research is required to investigate the impact of circadian misalignment on operational outcomes during spaceflight. In addition, it would be prudent to test the effectiveness of countermeasures, such as new lighting design, on the ISS prior to deploying such systems for longduration space missions.

\section{MATERIALS AND METHODS}

All astronauts selected for long-duration spaceflight from Expedition 14 (2006) until Expedition 26 (2011) were invited to participate in the study. Due to National Aeronautics and Space Administration (NASA) operational constraints on crew time, we were limited to a sample size of 21 participants. The first 21 volunteers to provide informed consent and complete spaceflight were included in the study. The protocol was approved by the Partners Healthcare Institutional Review Board and the NASA Committee for the Protection of Human Subjects.

Circadian phase estimates were calculated for data collected 11 days prior to launch (preflight) and throughout a spaceflight mission (spaceflight). Study participants wore wrist-borne actiwatches throughout each data collection block (Actiwatch-L; Minimitter-Respironics, Phillips Healthcare, Bend, OR, USA) providing sleep/wake activity and light exposure intensity in 2-min epochs in flight and 1-min epochs during preflight. A daily sleep log-including bedtime, wake time, number and cause of awakenings, ratings of sleep quality (ranging from 0 to 100 on a visual analog scale) and medication, and caffeine use-was completed continuously during the preflight interval and for $\sim 1$ week out of every 3 weeks while in space. For these analyses, we categorized medication use as sleep medication use, alertness medication use, and other medication use. There were no restrictions on behavior during data collection.

Information on timing of extra vehicular activities (EVAs, space walks), vehicle launch, and time of docking/undocking with ISS was obtained from NASA mission records.

\section{Circadian performance simulation software}

Circadian Performance Simulation Software (CPSS version 2.1, Brigham and Women's Hospital, Boston, MA, USA), designed to predict the effects of sleep-wake schedules and light exposure on the human circadian pacemaker, was used to estimate circadian phase for all participants for the preflight and spaceflight data collection intervals. This software was developed based on bio-mathematical models developed and refined at Harvard over many years. ${ }^{47-51}$ CPSS has been used to aid in timing light exposure sessions in laboratory and field studies and in each of these cases, the performance and circadian phase predictions generated by CPSS were consistent with objective circadian phase and performance markers. ${ }^{29,52,53}$ Sleep-wake (binary) and light exposure data (binned in $1 \mathrm{~h}$ increments) collected from the Actiwatch-L were used as inputs in the CPSS model. The astronauts were instructed to keep the actiwatch uncovered at all times. Details on how sleep duration was scored are described elsewhere. ${ }^{\prime}$ All available complete 24-h intervals of actigraphy data were included in the analysis. During episodes when the actiwatch was removed (e.g., during EVA), the lighting and sleep-wake data from the prior day were carried forward (4.9\% of days). Whenever the actiwatch was removed for more than 7 consecutive days, all data following actiwatch removal were excluded from the analysis (11.7\% of days).

The daily estimated endogenous circadian temperature minimum obtained from the CPSS program was compared with the sleep episode time derived from actigraphy and photometry for that day. When the estimated endogenous circadian temperature minimum fell within the sleep episode, that day's sleep episode was considered to be circadian "aligned." When the estimated endogenous circadian temperature minimum fell outside the sleep episode, that day's sleep episode was considered to be circadian "misaligned." The frequency of aligned and misaligned sleep episodes was quantified during preflight, spaceflight, for the sleep episodes immediately prior to EVAs, and during intervals when a vehicle was docked with ISS. Sleep outcomes and medication use were compared between aligned and misaligned conditions.

\section{Statistical methods}

Univariate summary statistics were calculated for all participants in order to evaluate population characteristics. Tests of significance for binary outcomes were computed using $x^{2}$-tests. For comparisons of sleep outcomes between participants by estimated endogenous circadian temperature minimum being aligned or misaligned, mixed-effects regression models to account for repeated measures were fitted to the sleep outcomes of interest. In these analyses, sleep episode was included as a fixed effect and participant was included as a random effect. All outcome values are presented as means and s.d. unless otherwise specified in the text.

\section{ACKNOWLEDGMENTS}

We thank the Shuttle and ISS crewmembers who participated in this study; Eymard Riel; Mia Jacobsen; Lauren Brogna, Liza Burke and Sean Benedix for data archiving and analysis; Elizabeth Klerman and Scott Beckett for technical support; NASA, Lockheed Martin and Wyle, for logistical support, especially, Marty Bost, Curtis Kershner, Scott Humbert, Karen Lawrence, Pam Baskin, Kristine Ohnesorge, and Lauran Johnson; and John Charles, Smith L Johnston, Lauren B Leveton, and Alexandra Whitmire and Suzanne McCollum, and Laura Bollweg at NASA Johnson Space Center for research support. The study was supported by NASA cooperative agreement NCC 9-119. Barger and Flynn-Evans were the recipients of National Heart, Lung and Blood Institute fellowships in the program of training in Sleep, Circadian and Respiratory Neurobiology at Brigham and Women's Hospital (NHLBl; T32 HL079010). Czeisler is the incumbent of an endowed professorship provided to the Harvard Medical School by Cephalon.

\section{CONTRIBUTIONS}

EEF-E, LKB and CAC conceived, designed, and supervised the study. EEF-E and LKB obtained the data. All authors analyzed and interpreted the data, critically revised the manuscript for important intellectual content, and provided administrative, technical, or material support. LKB, EEF-E, and CAC drafted the manuscript.

\section{COMPETING INTERESTS}

LKB has previously received research support from Cephalon, NFL charities, Sysco and San Francisco Bar Pilots. She has received consulting/lecture fees or served as a board member for Alertness Solution, Ceridian, Davis Joint Unified School Board, San Jose State University Foundation, Pugot Sound Pilots, Sygma and Torvec. EEF-E is an employee of NASA Ames Research Center; AAK has a patent, REM-Sleep Directed Visual Alarm System and Method, issued. CAC has received consulting fees from, or served as a paid member of scientific advisory boards for Amazon.com, Inc., A2Z Development Center, Inc., Bose Corporation, Boston Red Sox, Cleveland Browns, Institute of Digital Media and Child Development, Jazz Pharmaceuticals, Inc., Koninklijke Philips Electronics, Merck Sharpe and Dohme, Novartis, Purdue Pharma, Quest Diagnostics, Samsung Electronics, Teva Pharmaceutical Industries, and Vanda Pharmaceuticals; owns an equity interest in Somnus and Vanda Pharmaceuticals; and receives research/education support from Philips Respironics, Mary Ann and Stanley Snider through Combined Jewish Philanthropies, National Football League Charities, Optum, San Francisco Bar Pilots, Schneider National, Simmons, Sysco. CAC receives royalties from McGraw Hill, Houghton Mifflin Harcourt Philips and from Respironics for the Actiwatch-2 and Actiwatch-Spectrum devices used in this research. $C A C^{\prime}$ 's interests were reviewed and are managed by Brigham and Women's Hospital and Partners HealthCare in accordance with their conflict of interest policies. The Harvard Medical School, Division of Sleep Medicine and the Brigham and Women's Hospital, Division of Sleep and Circadian Disorders (LKB, EEF-E, CAC) have received unrestricted educational gifts and endowment funds from Boehringer Ingelheim Pharmaceuticals, Inc., Cephalon, Takeda Pharmaceuticals, Sanofi-Aventis, Tempur-Pedic, Walmart, Proctor and Gamble, Optum, Bose, Vanda Pharmaceuticals, Inc., Apria Healthcare, ResMed Foundation, Teva Pharmaceutical Industries, Ltd., Wake Up Narcolepsy, and Sepracor. CAC is the incumbent of an endowed professorship provided to Harvard University by Cephalon, and holds several process patents in the specialty of sleep and circadian rhythms (e.g., photic resetting of the human circadian pacemaker). CAC has served as an expert witness on various legal cases related to Celadon, Crete Carrier Corporation, Citgo, sleep and circadian rhythms, including matters related to Celadon, Crete Carrier Corporation, Citgo, Bombardier, Fedex, HG Energy, Michael Jackson's mother and children, Purdue Pharma, Stric Lan LLC, Valero, and United Parcel Service. 


\section{REFERENCES}

1. Barger, L. K. et al. Prevalence of sleep deficiency and use of hypnotic drugs in astronauts before, during, and after spaceflight: an observational study. Lancet Neurol. 13, 904-912 (2014).

2. Dijk, D. J. et al. Sleep, performance, circadian rhythms, and light-dark cycles during two space shuttle flights. Am. J. Physiol. Regul. Integr. Comp. Physiol. 281, R1647-R1664 (2001).

3. Gundel, A., Polyakov, V. V. \& Zulley, J. The alteration of human sleep and circadian rhythms during spaceflight. J. Sleep Res. 6, 1-8 (1997).

4. Monk, T. H., Buysse, D. J., Billy, B. D., Kennedy, K. S. \& Willrich, L. M. Sleep and circadian rhythms in four orbiting astronauts. J. Biol. Rhythms 13, 188-201 (1998).

5. Santy, P. A., Kapanka, H., Davis, J. R. \& Stewart, D. F. Analysis of sleep on Shuttle missions. Aviat. Space Environ. Med. 59, 1094-1097 (1988).

6. Czeisler, C. A. et al. Stability, precision, and near-24-hour period of the human circadian pacemaker. Science 248, 2177-2181 (1999).

7. Santhi, N., Horowitz, T. S., Duffy, J. F. \& Czeisler, C. A. Acute sleep deprivation and circadian misalignment associated with transition onto the first night of work impairs visual selective attention. PLoS ONE 2, e1233 (2007).

8. Flynn-Evans E. E., Gregory K., Arsintescu L., Whitmire A., Leveton L. B. Risk of performance decrements and adverse health outcomes resulting from sleep loss, circadian desynchronization, and work overload in human health and performance risks of space exploration missions NASA human research roadmap (http://humanresearchroadmap.nasa.gov/evidence/reports/sleep.pdf) (2015).

9. Hooke, L. R., Radtke, M., Garshnek, V., Teeter, R. \& Rowe, J. E. NASA Contractor Report USSR Space Life Sciences Digest 1986; 3822(08), Washington, DC.

10. Chang, A. M., Aeschbach, D., Duffy, J. F. \& Czeisler, C. A. Evening use of lightemitting eReaders negatively affects sleep, circadian timing, and next-morning alertness. Proc. Natl Acad. Sci. USA 112, 1232-1237 (2015).

11. Monk, T. H., Kennedy, K. S., Rose, L. R. \& Linenger, J. M. Decreased human circadian pacemaker influence after 100 days in space: a case study. Psychosom. Med. 63, 881-885 (2001).

12. Czeisler, C. A., Chiasera, A. J. \& Duffy, J. F. Research on sleep, circadian rhythms and aging: applications to manned spaceflight. Exp. Gerontol. 26, 217-232 (1991).

13. McMenamin, T. M. A time to work: recent trends in shift work and flexible schedules. Bureau of Labor Statistics 2007; Washington, DC.

14. Czeisler, C. A. Duration, timing and quality of sleep are each vital for health, performance and safety. Sleep Health 1, 5-8 (2015).

15. Buxton, O. M. et al. Adverse metabolic consequences in humans of prolonged sleep restriction combined with circadian disruption. Sci. Transl. Med. 4, 129ra143 (2012).

16. Tenkanen, L., Sjoblom, T., Kalimo, R., Alikoski, T. \& Harma, M. Shift work, occupation and coronary heart disease over 6 years of follow-up in the Helsinki Heart Study. Scand. J. Work Environ. Health 23, 257-265 (1997).

17. Stevens, R. G. et al. Considerations of circadian impact for defining 'shift work' in cancer studies: IARC Working Group Report. Occup. Environ. Med. 68, 154-162 (2011).

18. Flynn-Evans, E. E., Mucci, L., Stevens, R. G. \& Lockley, S. W. Shiftwork and prostatespecific antigen in the National Health and Nutrition Examination Survey. J. Natl Cancer Inst. 105, 1292-1297 (2013).

19. Lockley, S. W. et al. Effect of reducing interns' weekly work hours on sleep and attentional failures. N. Engl. J. Med. 351, 1829-1837 (2004).

20. Landrigan, C. P. et al. Effect of reducing interns' work hours on serious medical errors in intensive care units. N. Engl. J. Med. 351, 1838-1848 (2004).

21. Barger, L. K. et al. Extended work shifts and the risk of motor vehicle crashes among interns. N. Engl. J. Med. 352, 125-134 (2005).

22. Lindbeck S., Adasiak A., Richter C. K., Spivey P. SPILL The wreck of the Exxon Valdez. Alaska Oil Spill Commission, 1990.

23. Report of the Presidential Commission on the Space Shuttle Challenger Accident II. Appendix G. (http://history.nasa.gov/rogersrep/v1comm.htm\#staff) US Government Printing Office, Washington, DC, 1986.

24. Arthur, C. Mir crash `arose from Russian spending cuts. (http://www.independent. co.uk/news/mir-crash-arose-from-russian-spending-cuts-cuts-caused-mir-crashwith-1182424.html) London, UK (1998).

25. Board Meeting: Crash of Asiana Flight 214 Accident Report Summary. National Transportation and Safety Board (2014).

26. Kelly, S. M. et al. Flight controller alertness and performance during spaceflight shiftwork operations. Hum. Perf. Extrem. Environ. 3, 100-106 (1998).

27. Barger, L. K., Sullivan, J. P., Bollweg, L. J., Lockley, S. W. \& Czeisler, C. A. Experimental trial of fatigue countermeasure program in operational flight controllers. Human Research Program Investigators' Workshop (Houston, TX, 2013).

28. Wright, K. P. Jr., Hull, J. T., Hughes, R. J., Ronda, J. M. \& Czeisler, C. A. Sleep and wakefulness out of phase with internal biological time impairs learning in humans. J. Cogn. Neurosci. 18, 508-521 (2006).
29. Gronfier, C., Wright, K. P. Jr., Kronauer, R. E. \& Czeisler, C. A. Entrainment of the human circadian pacemaker to longer-than-24-h days. Proc. Natl Acad. Sci. USA 104, 9081-9086 (2007)

30. Basner, M. et al. Mars 520-d mission simulation reveals protracted crew hypokinesis and alterations of sleep duration and timing. Proc. Natl Acad. Sci. USA 110, 2635-2640 (2013).

31. Wotring, V. E. Medication use by U.S. crewmembers on the International Space Station. FASEB J. 29, 4417-4423 (2015).

32. Czeisler, C. A., Moore-Ede, M. C. \& Coleman, R. H. Rotating shift work schedules that disrupt sleep are improved by applying circadian principles. Science $\mathbf{2 1 7}$, 460-463 (1982).

33. Ursin, H., Endresen, I. M. \& Ursin, G. Psychological factors and self-reports of muscle pain. Eur. J. Appl. Physiol. Occup. Physiol. 57, 282-290 (1988).

34. Czeisler, C. A. et al. Exposure to bright light and darkness to treat physiologic maladaptation to night work. N. Engl. J. Med. 322, 1253-1259 (1990).

35. Horowitz, T. S., Cade, B. E., Wolfe, J. M. \& Czeisler, C. A. Efficacy of bright light and sleep/darkness scheduling in alleviating circadian maladaptation to night work. Am. J. Physiol. Endocrinol. Metab. 281, E384-E391 (2001).

36. Hanifin, J. P. \& Brainard, G. C. Photoreception for circadian, neuroendocrine, and neurobehavioral regulation. J. Physiol. Anthropol. 26, 87-94 (2007).

37. Lockley, S. W. et al. Short-wavelength sensitivity for the direct effects of light on alertness, vigilance, and the waking electroencephalogram in humans. Sleep 29, 161-168 (2006).

38. Rahman, S. A. et al. Diurnal spectral sensitivity of the acute alerting effects of light. Sleep 37, 271-281 (2014).

39. Whitson, P. A., Putcha, L., Chen, Y. M. \& Baker, E. Melatonin and cortisol assessment of circadian shifts in astronauts before flight. J. Pineal Res. 18, 141-147 (1995).

40. Dijk, D. J. et al. Amplitude reduction and phase shifts of melatonin, cortisol and other circadian rhythms after a gradual advance of sleep and light exposure in humans. PLoS ONE 7, e30037 (2012).

41. Viola, A. U., James, L. M., Schlangen, L. J. \& Dijk, D. J. Blue-enriched white light in the workplace improves self-reported alertness, performance and sleep quality. Scand. J. Work. Environ. Health 34, 297-306 (2008).

42. Brainard, G. C. et al. Solid-state lighting for the International Space Station: tests of visual performance and melatonin regulation. Acta Astronautica 92(1): 21-28 (2013).

43. NASA ISS SSLA Specificiation, Revision C., Johnson Space Center S684-13489 (2013).

44. Brigham and Women's Hospital BWH Bulletin. BWHers Win NASA Innovation Award for Space Station Lighting (http://www.brighamandwomens. org/about_bwh/publicaffairs/news/publications/DisplayBulletin.aspx?articleid=6528) (2015).

45. Jewett, M. E. \& Kronauer, R. E. Interactive mathematical models of subjective alertness and cognitive throughput in humans. J. Biol. Rhythms 14, 588-597 (1999).

46. Strogatz, S. H., Kronauer, R. E. \& Czeisler, C. A. Circadian pacemaker interferes with sleep onset at specific times each day: role in insomnia. Am. J. Physiol. 253, R172-R178 (1987).

47. Jewett, M. E., Forger, D. B. \& Kronauer, R. E. Revised limit cycle oscillator model of human circadian pacemaker. J. Biol. Rhythms (1999); 14, 493-499.

48. Kronauer, R. E., Forger, D. B. \& Jewett, M. E. Quantifying human circadian pacemaker response to brief, extended, and repeated light stimuli over the phototopic range. J. Biol. Rhythms (1999); 14, 500-515.

49. Jewett, M. E., Kronauer, R. E. \& Czeisler, C. A. Phase-amplitude resetting of the human circadian pacemaker via bright light: a further analysis. J. Biol. Rhythms (1994); 9, 295-314.

50. Jewett, M. E. et al. Human circadian pacemaker is sensitive to light throughout subjective day without evidence of transients. Am. J. Physiol. (1997); 273, R1800-R1809.

51. Jewett, M. E. \& Kronauer, R. E. Refinement of a limit cycle oscillator model of the effects of light on the human circadian pacemaker. J. Theor. Biol. (1998); 192, 455-465.

52. McKenna L. M. Sleep-wake actigraphy and light exposure during the Phoenix Mars Lander study. Master's Thesis, University College Dublin (2010).

53. Barger, L. K. et al. Learning to live on a Mars day: fatigue countermeasures during the Phoenix Mars Lander mission. Sleep 35, 1423-1435 (2012).

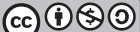

This work is licensed under a Creative Commons AttributionNonCommercial-ShareAlike 4.0 International License. The images or other third party material in this article are included in the article's Creative Commons license, unless indicated otherwise in the credit line; if the material is not included under the Creative Commons license, users will need to obtain permission from the license holder to reproduce the material. To view a copy of this license, visit http:// creativecommons.org/licenses/by-nc-sa/4.0/ 